\title{
Diferencias sexuales en los patrones de uso de Internet en una muestra de estudiantes de bibliotecología en una universidad peruana
}

\author{
Carlos ManUel VÍlCHEZ ROMÁN ${ }^{1}$ \\ Universidad Nacio nal Ma yor de San Marcos (UNMSM). \\ E-mail:adm1@viabcp.com
}

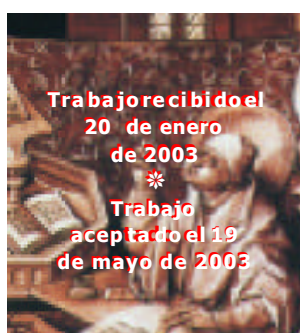

\begin{abstract}
RESUMEN
Se uti li zó una en cues ta de opi nión en una mues tra de 101 es tu dian tes de pregra do (44 va ro nes y 57 mu je res) de la es pe cia li dad de bi blio te co lo gía y ciencias de lain for ma ción de una univer sidad pe rua na, con el propó si to deiden tificar, según el sexo de los en cues ta dos, los pa tro nes de uso de In ter net y de ana li zar la per cep ción de los es tu dian tes so bre su de sem pe ño fren te a las herramientas ofrecidas por la red. Para estudiar la percepción sobre ésta se adap tó el ins tru men to di se ña do por Ford y Miller(1996). En tre los prin cipa les re sul ta dos se des ta can los siguien tes: 1) no se en con tra ron di fe ren cias signi fica tivas en tre hom bres y mu je res en cuan to a los pa tro nes de uso de In ternet.2)ElAnálisis deComponen tes Principales (ACP)permitióidentificarcincoconductas de uso de In ternet, locual permitió ca rac te rizarla ma ne racomo el usua rio abor da la In ter net con base en su ca pa ci dad de ma ne jo y la utilidad percibidadelasherramien tas y servicios que proporcionala In ternet. Al final se analizan estos resultados a la luz de los hallazgos de estudios previos, se discuten las implicancias de estos resultados y se planteanrecomendaciones para hacer estudios futuros.
\end{abstract}

Palabras Clave:Diferenciasse xuales, Bús que dade infor mación, Patrones de usodelnternet

\section{GENDER DIFFERENCES IN THE PATTERNS OF INTERNET USAGE, IN A SAMPLE OF LIBRARY SCIENCE STUDENTS OF A PERUVIAN UNIVERSITY CAR los MANUEL VÍlCHEZ-ROMÁN}

\begin{abstract}
A sur vey of opin ion in a sam ple of 101 un der gradu ate stu dents (44 males and 57 females) of Library and In forma tion Science was made in a Peru vian university. Its pur pose was to iden tify the pat terns of use of Inter net ac cord ing to the
\end{abstract}

1 Elau torde sea agra decerla cola bo ra ción delinves tiga dorso cial Arís tides Vara Homa en el análi sis estadístico de los datos e interpretación de los resultados, y también al bibliotecólogo Orlando CorzoCauracuri por las observaciones y sugerenciasbrindadas. 
gender of the participants, and to analyze the perception of their own per form ance in re la tion to the tools of fered to them by the WEB. In order to do this the in stru ment de signed for Ford and Miller (1966) was adapted. Main results are here listed: 1) No significant differences were found between men and women as to pat terns of Inter net us age. 2) Analy sis of main components (AMC) al lowed iden ti fica tion of five be hav iors in Internetus age. This helped us to dis tin guish the way a user ap proaches Inter net based on his or her han dling ca pacity and their perceived use ful ness of the in struments Inter net pro vides. Re sults are also ana lyzed in the light of pre vi ous findings and implications are discussed for future studies.

Key Words: Sexual Differences, Information Search; Internet Use Patterns

\section{ANTECEDENTES}

Sehanpublicadonumerosos estudiosquedemuestranlaexisten cia dediferencias biológicas, neurológicas y psicológicasentrevarones y mujeres, ein ten tan explicar por qué ambos piensan, sienten y se comportan de modo totalmente distinto. Biólogos, neurólogos, médicosypsicólogoshanestudiadolos roles masculinosyfemeninos. Eltrabajo de es tos inves tiga do res ha produ cidoabun dan te eviden cia empíricaso breloque hace di fe ren tes alos se xos: los da tos bio ló gicos, fisioló gicos, bioquímicos, neurológicosypsicológicos revelanprofundas diferenciasentrelossexos (Arnold, 1980; Erwin et al., 1989; Haier y Benhow, 1995; Ki mu ra, 1987, 1992; Moir y Jessel, 1991; Saykin et al., 1995; Shaywitz et al., 1995; Watson, 1991; Witelson, 1976, 1989). Por ejemplo,Do reen Kimura (1992) en contró quelas habilidades cognitivas entrehombresymujeres reflejanundesarrollohormonaldife renciado, elcualafecta directamente la estructurayorganización del sistema nervioso central y del cerebro en particular. La investigadoracanadiense planteó que estas diferenciasrevelan habilidades y destrezas distintas en hombres y mujeres, independientemente del proceso de so cialización. Enese sentido, las carre rasqueen fatizanlasha bilidades matemáticas o espaciales (por ejemplo, la ingeniería o la física) tienen mayor presencia masculina, mien tras que en ca rre ras cen tra das en ha bi lida des verbales y de co mu nicación interpersonal, la presencia femenina es dominante.

Enun trabajopos te rior, los neu rólogos Sallyy Ben nettShaywitz, dela Universidad deYale, publicaronunes tu diosobrelas diferenciassexualescerebrales, cuyoanálisis se centró en elprocesamien to depalabras. Alosparticipantes en elexperimento seles pidióque aso cia ran las pala bras queri ma ban en tre sí, mien trasquelos in ves tiga do res regis trabanlaactividadcerebralgraciasalasimágenes ob tenidas vía reso nan cia magnética. De acuerdo con los resultados de la investigación, durante las tareas verbales las mujeres activan ambos hemisferios cerebrales y los hombres sólo hacen trabajar una pequeñaregióndelhemisferiocerebralizquierdo(Shaywitz etal., 1995). 
Enlosúltimosaños, inves tiga doresprocedentes de diversoscamposhanexplora do la for ma en que las di fe ren cias se xua les de los es tu dian tes in flu yen en el uso de la tecnología de la información durante el colegio (Eden y Hullbert, 1995; Gurain y Hen ley, 2001; Kraut, Scher lis, Mukho padhyay, Ma ning y Kies ler, 1996; Leong y AlHa wamdeh, 1999; Moir y Jessel, 1991), y su re la ción con los es ti los de bús que da de in forma ción por Interneten la etapa universitaria (Fordy Miller, 1996; Ford, Millery Moss, 2001). Mi chael Gu rain (2001), dela Universidad de Missouri, es tu dió los principios biológicos (léase, desa rrolloneurológicoy hormonal) queafectan elapren dizaje deniños y niñas du ran te la eta pa es colar des dela prima ria has tala se cun da ria, y encontró diferencias importantes en el estilo de aprendizaje de cada uno de ellos. Con rela ción alas ha bili da des de ma nejode in for ma ción, NigelFord y Da vidMiller (1996), de la Universidad de Sheffield, realizaron un estudio con 75 estudiantes de pregra doy postgrado, en el cual analizaron sus percepciones relaciona das con eluso deInternet.Deacuerdoconloshallazgosobtenidosporestosinves tigadoresbritánicos, los hombres y las mu je res bus canin forma ción y ex ploran el con tenido de In ternetdeformadiferente.

Comopuedeobservarse, losestudios realizadoscones tudiantesdecolegio(college) yuniversidad mues tran di feren cias marca das en la forma como hom bres y mujeresutilizanla tec nología delain formación para fines edu cativos. Con la finalidad de explicarestas diferencias, losinvestiga do reshan propuestodiversasrazones:ansiedad informática,experiencia previa en el hogar y capacitación recibida, entre otras. Sobrela primera de ellas se ha en con tra doquelas mujeres tienen niveles más altos de ansie dad in formática quelos varones (Bros nan, 1998). Con rela ciónala ex periencia previa, Gurain (2001) encontró que los niños son alentados, permanentemente, a buscar y usar computadoras y, además, suelen manifestar su deseo de utilizarlas de for ma más vehe men te que las niñas, pues ellas tien den a de jar que sus com pa ñe ros dominen el tiem po de uso delas com pu ta doras. Alpa re cer, el es tímulo visual (léa se, interfaz grá fica) de las pan ta llas se ade cua me jor a los ni ños, quie nes, en pro me dio, tienenhabilida desviso-espacialesmás desa rrolladas.Porsuparte,lasinvestigadoras Siew Chee Long y Suliman Al-Hawamdeh (1999), de la Universidad de Singapur, evaluaron la aplicación de un enfoque constructivista al proceso de enseñanza-aprendizaje en un curso de cien cias, utilizan do la web como pla ta formatec nológica. Al com pa rar el uso de las com pu ta do ras y de In terneten con tra ron que, en ge ne ral, los mu cha chos pa san más tiem po ju gan do con la com pu ta do ra en casa y tienen más ex perien cia en el uso delweb, mien tras quelas chicasprefie renlas lec ciones de cla sea tra vés delwebquelas lec ciones enlas au las tra dicionales. Estehallaz go reflejalac lara preferenciadelas es tu dian tes porla lec tu ra, sea en papelo en el mo nitordelacomputa do ra, cosa que no ocu rre con los mu cha chos. En ese sen tido, exis te am plia evidencia que sugiere la existencia de diferencias sexuales con relación al uso de Internet (Boneva, Kraut y Frohlich, 2001; Katz y Aspden, 1996; Kraut, Scherlis, Mukhopadhyay, Maning y Kiesler, 1996; Morahan-Martin y Schumacher, 1997; SchumacheryMorahan-Martin,1998). 
Otros es tu dios han ex plo ra do la re la ción en tre el sexo y el ma ne jo de las tec nologías de la información desde el llamado "enfoque de género" (Harris, 1999; Heimrathy Goulding,2001;Tarlin,1997).Porejemplo,lasinvestigadoras británicas Ro sie Heimrath y Anne Goul ding con si de ran que la poca aco gi da de las tec no lo gías de la información en tre las muje res se debe a un con di cio na mien to so cial que tien de a reforzares tereotiposse xuales (porejemplo "lascomputadoras son cosade hombres"). Según es tas inves tiga do ras, esto ex plica porquéla pro porción de alum nas matricula das en cur sos de com pu ta ción es me nor ala de los va ro nes, con lo cual la ex pe rien cia pre via con las com pu ta do ras tam bién se re du ce. Todo ello da lu gar a un círculo vi cio so que tien de a incrementar lo que ellas lla man "la bre cha de gé ne ro". Por su par te, la pro feso ra RomaHa rris haes tu diado, sobrela base deuna división sexualdeltrabajo, cómo la tecnología de la información transforma el trabajo bibliotecario. Concluye que dentro de este proceso de in nova ción tec no ló gi cala mujersueleserdeja da de lado. Así, deacuerdoconel "en fo que de géne ro", la menordes tre zain for mática delasmur jeres seexplicaporlaexistenciadeunamentalidad masculinaquetiendeareforzar los roles sexuales tradicionalesenlaeducación dehombres ymujeres, tan to en elcolegio comoenlauniversidadyenlosinstitutossuperiores.

Aun cuando al gunosinves tiga dores han afirma doqueelsexis modela cul turapatriar cal es la ra zón por la cual las mu je res no son tan há biles como los hom bres en el ma nejo decomputadoras, exis tenau to resquein formanno haberen contradoeviden ciasclaras desexismooactitudesdiscrimina toriashacialas mujeresquedeseanusarcomputadoras (Bozionelos, 1995, 1996; Gu rainy Henley,2001). Talcomolo sos tienela es pecialista en genética Ann Moir(MoiryMoir,2000), a pesar de quelas muje res encuen tran abu rridos lamayo ríadelosjuegosporcomputadora, yasea de es trategia (como AgeofEmpires y StarCraft) o de acción (como Doom y Quake), una vez que los programas informáticos se ade cuan asusinteresesnotienenproblemaalgunoparausarlas computa doras. Esteprocesoseobservacla ramente examinandocómohacambiadolacomposición demográft cadelosusuarios deInternet.Inicialmen teeraun terreno conunapresencia mayoritariamente masculina, pero en la actualidad la proporción de hombres y mujeres se va equilibrando, entre otras cosas porque la red ofrece espacios de comunicación como el correoelectrónico, las listas de interés y la mensajeríainstantánea, don dela des treza femeninaes, en promedio, superiorala delos varones (BonevayKraut,2001; Savicki, KelleyyLingenfelter,1996).

\section{OBJETIVOS}

Más que ex plo rarlos es te reo ti pos sexua les en tornoal uso delas tec no logías de la in formaciónyla comunica ción,enestainves tiga ción se haopta do porun es tudiodel tiempo de uso, las herramientas de Internet preferidas y la conducta de uso de este medio.Acontinuación, seenumeranlosobjetivosperseguidos: 
1. Iden ti fi carlos pa trones de uso de In ternet de los es tu dian tes de pre gra do en una universidad peruana.

2. Analizar la percepción de los estudiantes sobre su desempeño frente a las herramientas ofrecidas por Internet.

Se parte de la hipótesis de que existen diferencias sexuales entre los estudiantes univer sitarios con rela ción a los pa trones yla conduc ta deuso frentea In ternet, dado quela ma yo ría delos es tu dios pre vios ofre ce eviden cia a favor. No obs tan te, se debe señalar que estos estudios se han realizado en países donde el gobierno impulsa de forma directa el acceso público a Internet. En ese sentido los estudiantes de países como Estados Unidos, GranBretañaoSingapur,tienen mayorpo sibilidaddeaccederaIn ternetquelos es tu dian tes pe rua nos. En elPerú, más del $70 \%$ de los pe rua nos que ac ce de a In ter net lo hace a tra vés de las ca bi nas pú blicas (Apo yo, 2002); sinembargo, se tra tade unainicia tiva priva da que su pone un pago porparte delos usuarios, cosaquenoocurreenlospaísesanteriormentecitados.

\section{METODOLOGÍA}

\section{Cuestionario}

Se utilizó una encuesta de opinión aplicada a una muestra de 101 estudiantes de pregrado de la especialidad de bibliotecología y ciencia de la in forma ción de la Universi dad Na cio nalMa yordeSan Marcos (UNMSM). La mues tra se tomó de una poblaciónde 377 alumnos matricula dosen elprimersemestre delaño 2002. Portratarse deun es tu dio so bre la percep ción quelos es tu dian tes tienen de su de sem peño en Internetseutilizóesteinstrumentodeau torreporteporquepermiterecogerunaamplia va rie dad de da tos y es ta ble cerrela ciones en trelasva riables analiza das. Ensuversión final elcues tionario "Encues ta deopi nión para universita rios" es tuvo con forma do porcincoáreas:in forma ción demo grá fica, ex perien ciadeuso deIn ternet, preferencias, utilidadpercibidayconductadeusodeInternet.

\section{Información demográfica de los universitarios}

En esta sec ción se in clu ye ron tres va ria bles: sexo, edad y año de es tu dios. Para el análisis demográficoseutilizaronmedidasdetendenciacentral.Portratarsedeun estudio sobre di fe rencias sexuales, lava riablesexo fue usa da comopun to de cor tepara realizarlosanálisisestadísticos.

\section{Experiencia de uso}

Se in clu ye ron tres va ria bles que me dían la ex pe rien cia pre via con In ternet:horas de uso por se ma na, ho ras de uso por se sión y años de uso de In ter net. Para el análi sis deestasvariablestambiénseutilizaronmedidasdetendenciacentral.Elpropósitode 
esta sec ción fue iden ti fi car po si bles re la cio nes en tre la con duc ta de uso y el tiem po dedicadoaInternet.

\section{Preferencias}

Esteru bro es tuvo com pues to pordos variables: herramien tadeIn ternet pre ferida (correo electrónico, chat/messenger, web y telefonía por Internet) y razones para usar Inter net (por ejem plo, sa berlas no ti cias, co no cerper so nas, ha cerne go cios, et cétera).

\section{Utilidad}

Sólo se incluyó una variable en esta sección: utilidad percibida de Internet. Esta variablefue propuestaparaconocersiInternetles re sultabaútilpara el trabajo,los estudiosoladiversión(entretenimiento).

\section{Percepción y actitudes}

Para es tu diar la per cep ción so bre la con duc ta de uso de In ter net se adap tó el instrumento diseñado por Ford y Miller (1996) "Percepciones sobre Internet", que con tenía 12 pregun tas en for ma to Likertde 5 pun tos. Para el presen tees tu dio, se tra bajóconítems en forma to Li kertde 4 pun tos, los cuales me dían el grado de acuerdoo de sacuerdoconunase rie de enunciadoscen trados enlaconduc tade usodeInternet. Las variables incluidas en esta sección fueron: Reviso los lugares recomendados; Siempre hay solu ción; Es raro en con traralgoútil; Meman ten go en fo ca do; Ma nejo dein forma ciónirrelevante;Bus carmásquenavegar;Demasia dodesordenado;Prefie rolos íco nos ygrá ficos; Ten go poco do minio; Es me jor una visión glo bal; Tien do a perderme, y Es me jor te nerun plan. Para ana lizar la vali dezy con fia bi li dad de esta escala se utilizaron el Análisis de Componentes Principales (ACP) y el coeficiente AlfadeCronbach, respectivamente.

El instrumento de recolección de datos se aplicó entre el 24 y el 28 de junio de 2002. Para elllena dodelcuestionario cada en cues tadorlepidióales tudian tecolaborar con el es tu dio so brelas di feren cias se xua les en el uso de In ternet. La en cues ta se realizóenlossalonesdeclase.Eltiempopromediodellenadofuede8minutos.

\section{Muestra}

Se eligió la Universidad Nacional Mayor de San Marcos (UNMSM) porque concen tra más del $80 \%$ delos es tu dian tes de bi blio te co lo gíay cien cia delain formacióna nivel nacional, cifra que revela la importan ciaso cial delquehacerpro fe sio naldelos egresados de esta casa de es tu dios. Ade más, se tra ta ba de un gru po al que elinves tiga dor tenía acceso. Los miembros de la muestra estaban matriculados en los cursos de tecnologíadelain forma ciónII,Tecnologíadela Información III, y Análisisy di señode sis temas dein forma ciónII. Se de ci dió tra bajarcon es tos es tu dian tes porlanaturaleza 
de los cur sos en cues tión, los cua les gi ran en tor no al uso y apli ca ción de las tec no logíasinformáticas.

Dado que se trata de un primeracercamientoempíricoal temadelas diferencias sexualescon relaciónaluso deInternet,el presente es tudioesdetipodes criptivoy de nivelexploratorio.

\section{RESULTADOS Y DISCUSIÓN}

\section{Descripción de la muestra}

Del total de es tu dian tes in clui dos en la mues tra, 44 fue ron va ro nes y 57 mujeres, ci fra que man tu vola pro porción dela po bla ción es tu dian tilen la es peciali dad debibliotecología, donde se aprecia mayor presencia femenina. El rango de edad de los participan tes os ciló en trelos 18 ylos 29 años, yla edad pro me dio fue de 22 años (D.S. $=2.49$ años).

\section{Experiencia sobre uso de Internet}

Con relación a la experiencia sobre uso de Internet, se encontró que los varones usanInternetdu ran te más tiem poquelas mu jeres ( 9.4 horas fren te a 6.9 ho ras porsema na). Sin em bargo, tam bién se debe se ña larque, en am bos ca sos, la des via ción están dar fue muy alta ( 11.68 ho ras y 7.57 ho ras, res pec tiva men te) y no seen con tró que la diferenciafuera significativa $(t=1.339, g . l .=67.52, p=0.185)$, a pe sar de que los hombresusanInternetmástiempoquelasmujeres.

Esta va ria ción en el tiem po de uso re fle ja la am pli tud de te mas que lle va a los es tur diantes a usar Internet. Dado que se trata de estudiantes universitarios se esperaba encontrarunusoprincipalmente aca démicodelared, locual hubierapermitidoidentificarse siones deInternetconuna du ración promedio. Sinembargode acuerdocon las respuestas de los encuestados las razones para usar Internet son muy variadas: desde jugar en red y conocerpersonas hasta mantenerse al día en la carrera y buscar trabajo, aun que la mayor par te de las res pues tas re flejó unin te rés pores tarin formado, contar con información para los cursos de la universidad y satisfacer intereses perso nales. En ese sen tido, el tiem po de uso de la red no es igual si una personalee un artículo periodístico (buscando saber las noticias) que si participa de una conversación "en línea" (bus can do conocerpersonas). Esta va ria ción en el tiem po de usocrece aún más si se con si de ra la va ria ble sexo ya que, como se vio en la pri me ra par te del presente artículo, hombres y mujeres perciben, procesan y asimilan la información de forma dis tinta. Porelloes es pe ra bleque el tiem po de dica do ausarIn ternet (léa se, tiempoparaprocesarlainformación recibida) varíeentreun sexoyotro.

Re sul tadossimilaresse en con tra ron enlacantidad dehoras porse sióny en el to tal de años de uso deInternet. Noobs tan te en estaúl ti ma variable seobservómenordis persión en los re sul ta dos (D.S. = 1.64 años en los hombres y 1.51 años en las mujeres). Enelsiguien tegráficoseapreciamejorladistribucióndeestavariable. 

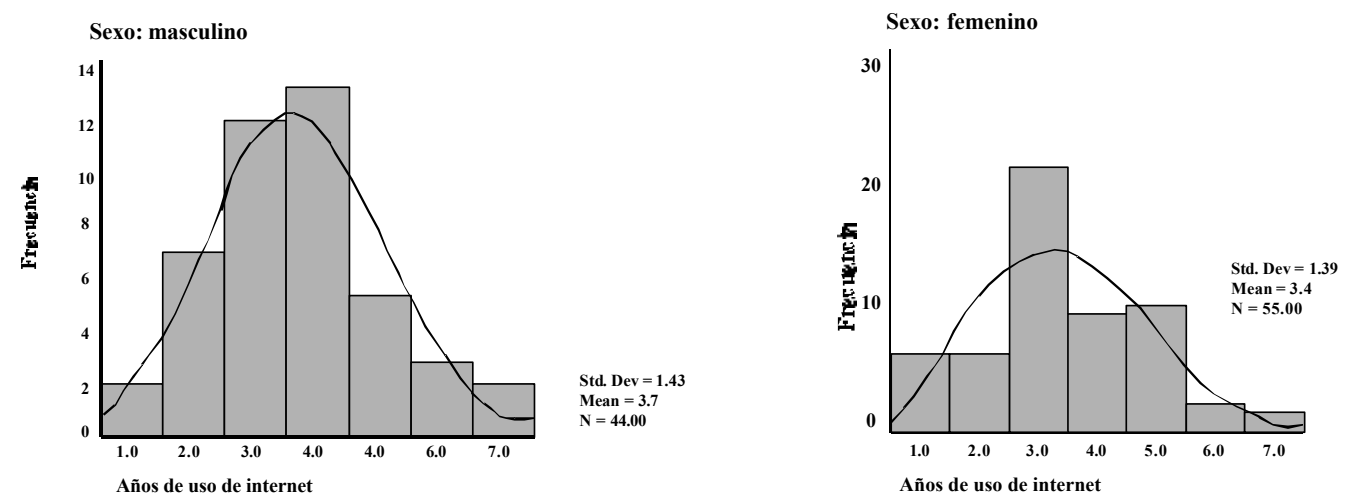

Gráfico 1

Años de uso de In ter net, según el sexo delen cuesta do

Aparen temen te se podría afirmarqueen estepun to exis te una "brechade géne ro" ya que los hombres em pie zan a usar In ternetan tes que las mu je res, pero si se ob servan los da tos con ma yor de te ni mien to se nota una proporción si milaren el nú me ro devaronesymuje resqueusaban In ternetan tes deen traralauniversidad ${ }^{2}(\mathrm{el} 23 \% \mathrm{de}$ cada sexo in for mó tener, al me nos, cin co años de ex pe rien cia en suuso). En ese sen tido, se puede considerar que, al menos en el caso de los estudiantes peruanos de biblio te co logía,la evidencia de una "brecha de géne ro" enel tiempode usodeIn ternet (léase, años de ex pe rien cia en el uso de la herra mien ta) no es tan con tun den te como sehabríaesperado.

\section{Herramientas preferidas}

Tan to hombres como mu jeres pre fie renusar, en primerlugar, elco rreo elec trónico $(69 \%$ en va ro nes y $60 \%$ en mu je res) y, como se gun da op ción, el World Wide Web $(53.7 \%$ en las muje res y $45.2 \%$ en los va ro nes). Eluso de he rra mien tas de co mu nicación en tiempo real (chat/messenger y telefonía por IP) tuvo baja aceptación. Nuevamente, se trata de diferencias estadísticas no significativas. Heimrath y Gouding (2001) llega ron a re sul ta dos simila res en sues tu diocualita tivo so brelapercepción y uso de Internet. De acuerdo con los resultados obtenidos por estas investigadoras,

2 Casila to ta lidad de los en cues ta dos se en con tra ba cursan do el $7^{\circ}$ y $8^{\circ}$ ci clo de la carre ra, es de cir, ya tenían cuatro años en la universidad cuando se realizóelestudio. 
hombresymujeresutilizanInternetenproporcionessimilares, básicamenteparacomunicarseatravésdelcorreoelectrónico.

Es interesanteobservar que aun cuan do las muje res tie nen, en pro me dio, ha bilidadesparalacomunicación oralyescrita más desarrolla das quelosvarones, sonellos quienes mues tran una preferencia mayor porel uso delco rreoelectrónico, unaherra mienta que básicamentesirve para comunicarse con otraspersonas. Se debeseñalar quese tra tadeun re sul ta do ines perado, pues de acuerdoconla li te ra tu ra revisada, se es perabaqueelcorreo electrónico fuerausa doen mayorproporción porlasmujeres; sinembargolosresultadosob tenidosrevelanlocontrario.Probablementeestoseexplicaporel nú me ro de usua rios va ro nes enla red, peroen elgru po de es tu dian tes universita riosencuestadoslas diferencias no fuerontanmarcadas. Portantoel resultado mereceserestudiadoconmayorprofundidad.

Con rela ción al World Wide Web, una he rra mien ta ba sa da prin ci pal men te en información textual (y en menor medida en elementos gráficos), es comprensible la mayor preferencia femenina, ya que para aprovechar esta herramienta es necesario leerlosartículos, es tu dios y documen tos publicadosen el weby, deacuerdocones tur dios realiza dos en años an te rio res, se sabe que las mu jeres tie nen me jor de sem peño lector que los va rones. Enese sen ti do, la pre fe ren cia femenina por el webven dría a ser consecuencia de su mejor desempeño en comprensión lectora. Es cierto que el atrac tivo del World Wide Web está en su as pec to vi sual, con imá genes y fo to gra fías agrada bles alavis ta.Sinem bargo, en términosgenerales, el apren dizaje (o re fuerzo) deconceptosyproce dimien tos, a niveluniversita rio, se da apartirdelcontenidot extual,nodelasimágenesy fotografías a color.

\section{Patrones de uso de Internet}

Sibien es cier to que se en con tra ron pa tro nes de uso dis tin tos en hom bres y mu jeres, no se halló evidencia empíricapara sostenerquese tra taba de diferenciases tadísticamentesignificativas. Enesesentido, las diferenciasobtenidassepodríanexplicar por la existencia de alguna variable subyacente con capacidad predictiva sobre el comportamiento de hom bres y muje res, másque porla parti cula ridad del mues treo realizado. El autor considera que esa variable latente es la edad de ini cio en el uso de consolas devideojuegos yde juegos porcomputa dora, ya queélhasidotes tigo delinterés mos tra do por ni ños y ado les cen tes en el uso de las con solas: en la ciu dad de Lima, en la década de los ochenta, los locales que ofrecían consolas de video (conocidas como pinballs) parajue gos deaccióneran vi si ta dos ex clu sivamente porlos alum nosde loscolegiosylasacademias preuniversitarias. ${ }^{3}$ En la dé ca da de los no ven ta, lospinballs fueron re em plaza dos porlas con solas Plays tationy Ninten do 32/64, pero el pú blico siguió sien do el mis mo. Esta di feren cia en la compo si ción delos usuarios adoles centes de los vi deo jue gos in du ce al au tor a con side rarla im por tan cia que asu mela edad

3 Centros de preparación para el ingreso a la universidad. 
de ini cio en el uso de es tos dis po si tivos elec tró nicos. A fin de ex plo raresta va ria ble con mayor profundidad en estudios posteriores se recomienda incluir un ítem que pregunteporlaedaddeinicioenelusodelasconsolas de videojuegos.

En la siguien te tablase pre sen ta los re sulta dos ob tenidos con relación a la Es cala de Con duc ta de uso y Opi nión so bre In ter net y se mues tra el por cen ta je de res puestas en cadaítem, se gúnelsexo delos encues tados.

Tabla 1

Conducta de uso y opinión sobre Internet, según sexo

\begin{tabular}{|c|c|c|c|c|}
\hline & $\begin{array}{l}\text { Totalmente en } \\
\text { desacuerdo }\end{array}$ & $\begin{array}{c}\text { En } \\
\text { desacuerdo }\end{array}$ & $\begin{array}{c}\text { De } \\
\text { acuerdo }\end{array}$ & $\begin{array}{l}\text { Totalmente } \\
\text { de acuerdo }\end{array}$ \\
\hline $\begin{array}{l}\text { Cuando uso Internetsólo } \\
\text { reviso las co sas que me } \\
\text { han recomendado. }\end{array}$ & $\begin{array}{l}\text { Hom bres: } 28.6 \% \\
\text { Mujeres: } 21.8 \%\end{array}$ & $\begin{array}{l}\text { Hom bres: } 54.8 \% \\
\text { Mujeres: } 50.9 \%\end{array}$ & $\begin{array}{l}\text { Hom bres: } 11.9 \% \\
\text { Mujeres: } 25.5 \%\end{array}$ & $\begin{array}{l}\text { Hom bres: } 4.8 \% \\
\text { Mujeres: } 1.8 \%\end{array}$ \\
\hline $\begin{array}{l}\text { Siempre en cuen trouna } \\
\text { forma ade cua da para utili- } \\
\text { zar In ter net de for ma } \\
\text { efectiva. }\end{array}$ & $\begin{array}{l}\text { Hom bres: } 4.5 \% \\
\text { Mujeres: } 5.4 \%\end{array}$ & $\begin{array}{l}\text { Hom bres: } 20.5 \% \\
\text { Mujeres: } 19.6 \%\end{array}$ & $\begin{array}{l}\text { Hom bres: } 56.8 \% \\
\text { Mujeres: } 60.7 \%\end{array}$ & $\begin{array}{l}\text { Hom bres: } 18.2 \% \\
\text { Mujeres: } 14.3 \%\end{array}$ \\
\hline $\begin{array}{l}\text { Rara vez en cuen tro algo } \\
\text { útil en In ter net. }\end{array}$ & $\begin{array}{l}\text { Hom bres: } 25.0 \% \\
\text { Mujeres: } 26.8 \%\end{array}$ & $\begin{array}{l}\text { Hom bres: } 54.5 \% \\
\text { Mujeres: } 53.6 \%\end{array}$ & $\begin{array}{l}\text { Hom bres: } 15.9 \% \\
\text { Mujeres: } 16.1 \%\end{array}$ & $\begin{array}{l}\text { Hom bres: } 4.5 \% \\
\text { Mujeres: } 3.6 \%\end{array}$ \\
\hline $\begin{array}{l}\text { Cuando es toy en In ternet } \\
\text { logro mantenermeen "el } \\
\text { obje tivo" y evito demasia } \\
\text { do materialirrelevante. }\end{array}$ & $\begin{array}{l}\text { Hom bres: } 6.8 \% \\
\text { Mujeres: }\end{array}$ & $\begin{array}{l}\text { Hom bres: } 29.5 \% \\
\text { Mujeres: } 37.7 \%\end{array}$ & $\begin{array}{l}\text { Hom bres: } 50.0 \% \\
\text { Mujeres: } 54.7 \%\end{array}$ & $\begin{array}{l}\text { Hom bres: } 13.6 \% \\
\text { Mujeres: } 7.5 \%\end{array}$ \\
\hline $\begin{array}{l}\text { Estoypreparado a hacerle } \\
\text { fren te a mu cha in for ma- } \\
\text { ción irre le van te si hay algo } \\
\text { útil en In ter net de lo cual } \\
\text { me podríaperder }\end{array}$ & $\begin{array}{l}\text { Hom bres: } 2.3 \% \\
\text { Mujeres: } 2.0 \%\end{array}$ & $\begin{array}{l}\text { Hom bres: } 27.3 \% \\
\text { Mujeres: } 25.5 \%\end{array}$ & $\begin{array}{l}\text { Hom bres: } 56.8 \% \\
\text { Mujeres: } 66.7 \%\end{array}$ & $\begin{array}{l}\text { Hom bres: } 13.6 \% \\
\text { Mujeres: } 5.9 \%\end{array}$ \\
\hline $\begin{array}{l}\text { Si tuviera que ele gir sola } \\
\text { men te uno, pre fe ri ría la } \\
\text { bús que da porpa la bras } \\
\text { que la ex plo ra ción/ na ve- } \\
\text { ga ción a tra vés de In ter- } \\
\text { net. }\end{array}$ & $\begin{array}{l}\text { Hom bres: } 4.8 \% \\
\text { Mujeres: } 7.1 \%\end{array}$ & $\begin{array}{l}\text { Hom bres: } 21.4 \% \\
\text { Mujeres: } 30.4 \%\end{array}$ & $\begin{array}{l}\text { Hom bres: } 54.8 \% \\
\text { Mujeres: } 50.0 \%\end{array}$ & $\begin{array}{l}\text { Hom bres: } 19.0 \% \\
\text { Mujeres: } 12.5 \%\end{array}$ \\
\hline $\begin{array}{l}\text { In ter net es de ma sia do de- } \\
\text { sorde na do para migus to. }\end{array}$ & $\begin{array}{l}\text { Hom bres: } 7.0 \% \\
\text { Mujeres: } 3.6 \%\end{array}$ & $\begin{array}{l}\text { Hom bres: } 27.9 \% \\
\text { Mujeres: } 30.4 \%\end{array}$ & $\begin{array}{l}\text { Hom bres: } 53.5 \% \\
\text { Mujeres: } 41.1 \%\end{array}$ & $\begin{array}{l}\text { Hom bres: } 11.6 \% \\
\text { Mujeres: } 25.0 \%\end{array}$ \\
\hline $\begin{array}{l}\text { Si al es tar en In ter net no } \\
\text { hi cie ra uso de íco nos y } \\
\text { grá fi cos yo no lo fre cuen- } \\
\text { taría tanto. }\end{array}$ & $\begin{array}{l}\text { Hom bres: } 9.3 \% \\
\text { Mujeres: } 9.3 \%\end{array}$ & $\begin{array}{l}\text { Hombres: } 44.2 \% \\
\text { Mujeres: } 38.9 \%\end{array}$ & $\begin{array}{l}\text { Hom bres: } 39.5 \% \\
\text { Mujeres: } 42.6 \%\end{array}$ & $\begin{array}{l}\text { Hom bres: } 7.0 \% \\
\text { Mujeres: } 9.3 \%\end{array}$ \\
\hline
\end{tabular}


Tabla 1

Conducta de uso y opinión sobre In ter net, según sexo (Cont.)

\begin{tabular}{|c|c|c|c|c|}
\hline & $\begin{array}{l}\text { Totalmente en } \\
\text { desacuerdo }\end{array}$ & $\begin{array}{c}\text { En } \\
\text { desacuerdo }\end{array}$ & $\begin{array}{c}\text { De } \\
\text { acuerdo }\end{array}$ & $\begin{array}{l}\text { Totalmente } \\
\text { de acuerdo }\end{array}$ \\
\hline $\begin{array}{l}\text { Considero que tengo } \\
\text { poco do mi nio para usar } \\
\text { Internet. }\end{array}$ & $\begin{array}{l}\text { Hom bres: } 22.7 \% \\
\text { Mujeres: } 18.2 \%\end{array}$ & $\begin{array}{l}\text { Hom bres: } 43.2 \% \\
\text { Mujeres: } 36.4 \%\end{array}$ & $\begin{array}{l}\text { Hombres: } 29.5 \% \\
\text { Mujeres: } 43 . \%\end{array}$ & $\begin{array}{l}\text { Hom bres: } 4.5 \% \\
\text { Mujeres: } 1.8 \%\end{array}$ \\
\hline $\begin{array}{l}\text { La me jor ma ne ra de usar } \\
\text { In ternet es ex plo rarde } \\
\text { todo am plia men tepara } \\
\text { te ner un pun to de vis ta } \\
\text { comparativosobre los di- } \\
\text { fe ren tes as pec tos y he rra } \\
\text { mien tas de In ter net, an tes } \\
\text { de do mi naral gu no con } \\
\text { profundidad. }\end{array}$ & $\begin{array}{l}\text { Hom bres: } 9.5 \% \\
\text { Mujeres: } 1.9 \%\end{array}$ & $\begin{array}{l}\text { Hom bres: } 33.3 \% \\
\text { Mujeres: } 21.2 \%\end{array}$ & $\begin{array}{l}\text { Hombres: } 40.5 \% \\
\text { Mujeres: } 65.4 \%\end{array}$ & $\begin{array}{l}\text { Hombres: } 16.7 \% \\
\text { Mujeres: } 11.5 \%\end{array}$ \\
\hline $\begin{array}{l}\text { Tien do a per der me cuan- } \\
\text { do uso In ter net. }\end{array}$ & $\begin{array}{l}\text { Hom bres: } 27.3 \% \\
\text { Mujeres: } 19.6 \%\end{array}$ & $\begin{array}{l}\text { Hom bres: } 38.6 \% \\
\text { Mujeres: } 48.2 \%\end{array}$ & $\begin{array}{l}\text { Hombres: } 29.5 \% \\
\text { Mujeres: } 32.1 \%\end{array}$ & $\begin{array}{l}\text { Hom bres: } 4.5 \% \\
\text { Mujeres: }\end{array}$ \\
\hline $\begin{array}{l}\text { Es me jor usar In ter net } \\
\text { sólo cuan do se tie ne un } \\
\text { plan bien definido,más } \\
\text { que an dar ex plo ran doy } \\
\text { navegando por distintos } \\
\text { lugares. }\end{array}$ & $\begin{array}{l}\text { Hom bres: } 9.3 \% \\
\text { Mu je res: } 12.5 \%\end{array}$ & $\begin{array}{l}\text { Hom bres: } 37.2 \% \\
\text { Mujeres: } 30.4 \%\end{array}$ & $\begin{array}{l}\text { Hom bres: } 34.9 \% \\
\text { Mujeres: } 39.3 \%\end{array}$ & $\begin{array}{l}\text { Hom bres: } 18.6 \% \\
\text { Mujeres: } 17.9 \%\end{array}$ \\
\hline
\end{tabular}

Seen contra ron di fe rencias marca das enlacon duc ta so bre uso deInternet. Engeneralenaquellas conductasasociadas aun manejoadecuadodeInternetloshombres mos tra ron una conduc ta más aser tiva, mien tras que en losítems liga dos a un manejo limitado de Internet las mujeres destacaron (sólo reviso los lugares recomendados, tengo poco dominio, etcétera). Estos resultados son consistentes con los hallazgos deFordy Miller(1996), quienes in formaron quelas muje res tien dena perderse enInternet, tie nen poco do minio de la he rra mien tay sólore visan los lu ga res re comendados, mien trasquelos hombres ex ploranInternetsinun plande finidoyen fren tangrandes volúmenes de información irrelevante con la esperanza de encontrar material quesatisfagasusinteresespersonales.

Con relaciónalítem "Prefe riríalabúsquedaporpalabrasqueexploración / navega ción a través de Internet”, una mayor proporción de varones se mostró de acuerdo (73.8\% devaronesy $62.5 \%$ de mujeres) con esta afir ma ción. Labús que da porpala bras supone el uso de los motores de búsqueda (search engines), herramientas que permiten ex plo rar el con teni do de un si tio web a tra vés de un pro gra ma que com pa ra los tér minos de bús que da in gre sa dos por el usua rio a tra vés de un for mu la rio de con sul ta, con 
las en tra das de unarchivoíndiceoarchivoinvertido. Portan to setrata deherramientas que exigen cier ta des tre za en el ma ne jo de progra mas, cam po en el quelos va ro nes suelen des ta car. Ford, Millery Moss (2001) in forman re sul ta dos simila res. En un es tudio realizado con 69 estudiantes de postgrado, ellos encontraron diferencias sexuales, estadísticamentesignificativas $(t=.2 .219 ; p=.030)$ en el es tilode bús que da de información. De acuerdo con sus resultados, al comparar las respuestas de hombres y mujeresseencontróquelos es tudiantesvarones emplea ronmás palabrasclave(keywords) alutilizarelmotordebúsquedaAltavista(3.35palabrasenpromedio).

En el modelo de regresiónlineal propues to pores tos inves tiga doresseencon tró que el sexomas culinoera un pre dic tor con fia ble de rele van cia enla re cupe ra ción de in forma ción (Ford, Millery Moss, 2001). En elpre sen tees tu diotambién seen con tró queloshombreslograronnivelesmásaltosderelevanciaautopercibida.

A fin de ver silas va ria bles de la Es ca la de con duc ta de uso y Opi nión so bre In ternetse agrupaban de algu na ma nerapar ticularse de cidió realizarun Análisis de Componentes Principales (ACP). Antes de ello se realizaron operaciones preliminares conla finalidad de ga ran tizarla validez de los re sul ta dos. En ese sen tido, los su puestos fue ronade cua dos (medidade con formidad Kaiser-Meyer-Olkin $=0.586 y$ test de esfericidad de Barlett $=142.199$, g.l. $=66, \mathrm{p} 0.0001)$. Una vez confirmados los supuestosseproce dióarealizarelAnálisis deComponentesPrincipales.Laex tracción de com ponen tes se de tu vo cuan do el va lor delEigenvalue no al can zóla uni dad (Eigenvalue 1). Este criteriopermitió ob tenerun to talde cin cocomponentes, los cuales fueron rota dos utilizan do elméto do or togo nal Varimax. Esta solu ción factorialde cinco componentes explicó la varianza total de los ítems en cantidades adecuadas $(20.8 \%, 14.2 \%, 10.8 \%, 10.2 \%$ y $8.7 \%$, res pec tiva mente). El porcen ta je to tal delavarianza acu mu la da de los cua tro com po nen tes fue de $56.54 \%$. En la tabla 2 se pre sentan es tos valo res, jun to conla dis tribu ción de losítems y sus res pec tivas cargas fac torialesenloscincocomponentesobtenidos.

Tabla 2

Distribución factorial y Eigen va lues delosítems en los cin co com po nen tes ob te ni dos

\begin{tabular}{|c|c|c|c|c|c|}
\hline \multirow[t]{2}{*}{ Ítems } & \multicolumn{5}{|c|}{ Componentes } \\
\hline & Comp. 1 & Comp. 2 & Comp. 3 & Comp. 4 & Comp. 5 \\
\hline $\begin{array}{l}\text { Cuando es toy en In ternetlogroman tenerme } \\
\text { en "el objetivo"y evitodemasiadomaterial } \\
\text { irrelevante. }\end{array}$ & 0.818 & & & & \\
\hline $\begin{array}{l}\text { Estoypreparado a ha cer le fren te a mu cha in- } \\
\text { formación irre le van te si hay algo útil en In- } \\
\text { ter net de lo cual me po dría per der. }\end{array}$ & 0.754 & & & & \\
\hline $\begin{array}{l}\text { Siem pre en cuen tro una for ma ade cua da para } \\
\text { utilizar In ter net de forma efec tiva. }\end{array}$ & 0.535 & & -0.438 & & \\
\hline Tien do a per der me cuan do uso In ter net. & & 0.821 & & & \\
\hline
\end{tabular}


Tabla 2

Distribución factorial y Eigenvalues de los ítems en los cincocomponentes obtenidos (Cont.)

\begin{tabular}{|c|c|c|c|c|c|}
\hline \multirow[t]{2}{*}{ Ítems } & \multicolumn{5}{|c|}{ Componentes } \\
\hline & Comp. 1 & Comp. 2 & Comp. 3 & Comp. 4 & Comp. 5 \\
\hline $\begin{array}{l}\text { Con si de ro que ten go poco do mi nio para } \\
\text { usar Internet. }\end{array}$ & & 0.821 & & & \\
\hline $\begin{array}{l}\text { Internetes de ma sia do de sordena do parami } \\
\text { gusto. }\end{array}$ & -0.409 & 0.424 & & & \\
\hline $\begin{array}{l}\text { Si tu vie ra que ele gir so la men te uno, pre fe ri ría } \\
\text { la bús que da por pa la bras que la ex plo ra- } \\
\text { ción/na vega ción a tra vés de In ternet. }\end{array}$ & & & 0.809 & & \\
\hline Rara vez encuentro algo útil en Internet. & & & 0.717 & & \\
\hline $\begin{array}{l}\text { Si al es tar en In ter net no hi cie ra uso de íco- } \\
\text { nos y grá fi cos yo no lo fre cuen ta ría tan to. }\end{array}$ & & & & 0.715 & \\
\hline $\begin{array}{l}\text { Cuan do uso In ter net sólo re vi so las co sas } \\
\text { quemehan recomen da do. }\end{array}$ & & & & 0.707 & \\
\hline $\begin{array}{l}\text { La me jor ma ne ra de usar In ter net es ex plo rar } \\
\text { de todo am plia men te para te ner un pun to de } \\
\text { vis ta compara tivo so brelos di feren tes as pec- } \\
\text { tos y he rra mien tas de In ter net, an tes de do- } \\
\text { minaralgunoconprofundidad. }\end{array}$ & & & & & 0.827 \\
\hline $\begin{array}{l}\text { Es me jor usar In ter net sólo cuan do se tie ne } \\
\text { un plan bien de fi ni do, más que an dar ex plo- } \\
\text { rando y navegando por distintoslugares. }\end{array}$ & & & & & 0.619 \\
\hline Eigenvalues & 2.49 & 1.70 & 1.30 & 1.22 & 1.04 \\
\hline Porcentajedevarianzaexplicada & 20.78 & 14.15 & 10.80 & 10.16 & 8.67 \\
\hline
\end{tabular}

$\mathrm{Al}$ analizar las cargas factoriales se identificaron cinco formas de aproximarse a Internet, las cuales iban desde un acercamiento de tipo experto hasta las personas que asumían una actitud bastante cautelosa frente a las herramientas y contenidos ofrecidos por la red. Por esta razón, cada componente o factor recibió un nombre queidentificaradeformaadecuadaalusuarioencuestión:

\section{C1 $\rightarrow$ Conocedores $\mathrm{C} 2 \rightarrow$ Novatos C3 $\rightarrow$ Atrevidos C4 $\rightarrow$ Ingenuos C5 $\rightarrow$ Metódicos}

Losconocedores siempreen cuen tranuna solu ciónalos problemasysabencómo manejarelvolumen dein formación disponibleenInternet. Precisamente ellosconsideran que la red no es de sordenada. Porsu parte, los nova tos tienen ha bilida deslimitadas para su manejoy, ade más, tienenunapercepciónnegativadelared(e.g.,ellos 
síconsideranquelared Internetes desordenada).Los atrevidostambiéntienenhabi lidades limi ta das pero asu men una ac ti tud más explora to ria;sinem bargo, no suelen tenertantoéxitocomolosconocedores (e.g. raravez encuentranuna forma efec tiva de usar Internet). Los ingenuos necesitanrecomendacióny guía para usar Internet. No es ca sual que losinge nuos mues tren una cla ra pre fe ren cia por lain ter fazgrá fica. Final mente, los me tó di cos son muy cau telosos al momen to de usarIn ternet. Esimportanteseñalarqueestos cincocomponentes (ofactores) sonindependientesentre sípueslascargas factorialesmuestranunaadecuadadistribución.

Como la hipótesis de la presente investigación señalaba la existencia de diferencias se xuales en trelos es tu dian tes universitarios con rela ción ala con duc ta deusode Internet, se decidió comparar las medias de los puntajes de hombres y mujeres en cada uno delos fac to res an te rior men te men cio na dos (C1, C2, C3, C4y C5). Para ello cada fac tor fue de finido como la suma arit mética delosítems con ma yor carga factorialendichocomponente, delasiguientemanera:

C1 = logro mantenerme en el objetivo + puedo manejar mucha información irrelevante + uso la red de forma efectiva

$\mathrm{C} 2$ = tiendo a perderme en Internet + tengo poco dominio para usar Internet + Internet es demasiado desordenada

C3 = prefiero la búsqueda por palabras que la exploración/navegación + rara vez encuentro algo útil en Internet

C4 = sino hu bieraíco nos y gráficos no usa ría tan to In ternet + sólo re vi solas co sas que me han recomendado

$\mathrm{C} 5=$ es mejor tenerun pun tode vis ta compara tivodelas herra mien tas + es mejor tener un plan global bien definido

En la tabla 3 sepresen taelpun tajepromedio ob tenido por hom bres ymuje res en cadaunodelosfactores.

Tabla 3

Puntaje promedio en cada uno de los factores, según sexo

\begin{tabular}{|l|c|c|}
\hline \multicolumn{1}{|c|}{ Factor } & Hombres & Mujeres \\
\hline $\mathrm{C} 1 \rightarrow$ Conocedores & 8.41 & 7.77 \\
\hline $\mathrm{C} 2 \rightarrow$ Novatos & 6.91 & 7.12 \\
\hline $\mathrm{C} 3 \rightarrow$ Atrevidos & 4.75 & 4.56 \\
\hline $\mathrm{C} 4 \rightarrow$ Ingenuos & 4.23 & 4.39 \\
\hline $\mathrm{C} 5 \rightarrow$ Metódicos & 5.09 & 5.19 \\
\hline
\end{tabular}


Estos resultados revelan una conducta de uso de Internet distinta en hombres y mujeres. Porunladolos varonessecaracterizan portenermayorconocimientodela red y ser más atre vi dos pero me nos me tó di cos que las mu je res, quie nes suelen mostrarmayorcautela pero tam bién tenerhabilida des más li mi ta das para el ma nejodeInternet. Sin em bargo se debe se ña lar que al aplicar la prue ba tde Stu dent para muestras independientes, seencon tró queestas diferenciasnoeranes ta dís ticamentesignificativas $(p=.09, p=.58, p=.50, p=.57$ y $p=.75)$. Esto pue de de ber se a la pre sen cia de una variablesubyacente ca paz de ex plicar es tas di fe ren cias en la con duc ta de uso de Internet.Alrespectootrasinvestigacioneshanidentificadodiferenciasestadísticamentesignificativas.

Por ejem plo, Ford, Millery Moss (2001), lue go de apli car un aná lisis de re gre sión múltipleen una mues trade es tu dian tes de postgrado, en con tra ron quelas fallas enla recu pe ra ción dela in forma ción es ta ban aso cia das con el sexo fe meni no y con la sensa ciónde notener elcon trolono poder evitarel ma terialirrelevante.Y, porel contrario, la recuperación exi to sa delain forma ción se rela ciona baconelsexo mas culino y conla sen sa ción de te ner el con troly de man tenerse en fo ca do en el ob je tivo du ran te lassesionesdeusodeInternet $(\mathrm{t}=2.434, p=.18 ; t=2.031 ; p=.47 ; t=-2.031 p=.47)$.

Losresultadosdeestees tudio también son consistentes conlos hallazgosidentificados por la psicóloga JanetMorahan-Martin y el investigador Phyllis Schumacher: las mujeres tienen más dificultad para encontrar información en línea y se sienten menossegu rascuan dousanlasherramien tas disponibles a través deInternet (Mora han-Martiny Schu ma cher, 1997; Schu ma cheryMo rahan-Martin,1998). Sinembar go, elestudioseñalaquelabre chain formáticaes menos pronunciadaenInternetque en eluso de com pu ta do ras en ge neral, de bido, en tre otras co sas, al uso in ten sivo del co rreoelectrónico porpartedelas mujeres, quienes mues tranunes tilodeco munica ción más expresivo, amiga bleycon máscontenidopersonalquelos va rones (Boneva y Kraut,2001;Herring,1994,1996; Sa vicki,KelleyyLingen felter, 1996).

\section{Confiabilidad de la Escala "Conducta de uso y opinión sobre Internet"}

La confiabilidad de esta escala se analizó utilizando el coeficiente Alfa de Cronbach.Estatécnicapermitecalcularlamagnitud delaconsis tenciainternadeunaescalay de terminarla so li dez de cadaítem den tro de ella, a tra vés del uso de dos in dica do res: correlación corregida ítem-test y valores del coeficiente Alfa de Cronbach si el ítem fue ra bo rra do, es de cir, si se eli mi na ra elítem de la esca la. A con ti nua ción, latabla4presentalosvaloresobtenidos. 
Tabla 4

Correlación corregidaítem-test y valores de Alfa si el ítem es borrado de la escala

\begin{tabular}{|c|c|c|}
\hline $\begin{array}{c}\text { Ítems de la escala "Conducta de uso y opinión } \\
\text { sobre Internet" }\end{array}$ & $\begin{array}{l}\text { Correlación co- } \\
\text { rregida ítem-test }\end{array}$ & $\begin{array}{l}\text { Alfa si el ítem } \\
\text { era borrado }\end{array}$ \\
\hline $\begin{array}{l}\text { Cuando uso In ter net sólo re vi so las co sas que me han re co- } \\
\text { mendado. }\end{array}$ & .2450 & .6041 \\
\hline $\begin{array}{l}\text { Siem pre en cuen tro una forma ade cua da para utilizar In ter net } \\
\text { deforma efectiva. }\end{array}$ & .1393 & .6218 \\
\hline Rara vez en cuen tro algo útil en In ter net. & .3043 & .5939 \\
\hline $\begin{array}{l}\text { Cuando es toy en In ter netlogro man te ner me en "elobje tivo" y } \\
\text { evitodemasiadomaterialirrelevante. }\end{array}$ & .2265 & .6076 \\
\hline $\begin{array}{l}\text { Estoypreparado a hacerle fren te a mu chain for ma ciónirrele- } \\
\text { vante si hay algo útil en Internet de lo cual me podríaperder. }\end{array}$ & .3195 & .5895 \\
\hline $\begin{array}{l}\text { Si tuviera que ele gir so la men te uno, pre fe ri ría la bús que da por } \\
\text { pala bras que la ex plo ra ción/na ve ga ción a tra vés de In ternet. }\end{array}$ & .3518 & .5829 \\
\hline Internetes dema sia do de sordena do para migus to. & .3499 & .5836 \\
\hline $\begin{array}{l}\text { Si al es tar en In ter net no hi cie ra uso de íco nos y grá fi cos yo no } \\
\text { lo frecuen ta ría tan to. }\end{array}$ & .3392 & .5855 \\
\hline Considero que ten go poco do mi nio para usar In ter net. & .2019 & .6121 \\
\hline $\begin{array}{l}\text { La me jor ma ne ra de usar In ter net es ex plo rar de todo am plia- } \\
\text { men te para te ner un pun to de vis ta com pa ra ti vo so bre los di fe- } \\
\text { rentes aspectos y herra mien tas deIn ternet, an tes de do minar } \\
\text { algunoconprofundidad. }\end{array}$ & .2521 & .6041 \\
\hline Tien do a per der me cuan do uso In ternet. & .1622 & .6183 \\
\hline $\begin{array}{l}\text { Es me jor usar In ter net sólo cuan do se tie ne un plan bien de fi- } \\
\text { nido, más que an dar ex plo ran do y na ve gan do por dis tin tos lut } \\
\text { gares. }\end{array}$ & .3210 & .5887 \\
\hline
\end{tabular}

De acuerdo con el análisis realizado, el valor Alfa de Cronbach para la escala "Conducta de uso y opinión sobre Internet" fue mo de ra damen te alto $(\alpha=0.6205)$. Estevalor ofre ció eviden cia de una solidez modera da en la con sis ten ciain terna dela escala. Enese sen tidoseobservaque sielsegun doítemes borrado, la consisten ciainternadelaes calasu biráa 0.6218. Además esteítempresen tauna débilcorrelacióncorregida ítem-test $(r=0.1393)$, lo cual refleja una capacidad limitada para discriminar en tre los pun ta jes al tos y ba jos de la es ca la. Pero si cual quie ra de los de másítems son borrados, el valor Alfa de Cronbach descenderá a menos de 0.6205. Esto significa que de los 12 ítems de la es cala, elítem nú me ro 2 es el menos con fiable. Para me jo rar la con sis ten cia in terna dela es ca la se re co mien da re visarlare dac ción de cada unode losítems, en particulardelítem nú mero 2 , y realizaruna adap ta ciónlingüís tica másr igu ro sa en es tu dios pos te rio res. Es tos cam bios po drían lo grarun nivel de con fia bili dad más alto; léa se unvalorde $\alpha=0.8$. 
Alaluzde es tosre sultadoscabepreguntarse sies tas diferencias noson productode un condicionamiento social que tiende a reforzar los roles sexuales tradicionales, tal como lo pos tu la el "en fo que de gé ne ro". Si bien es cier to que se tra ta de un tema que mereceunanálisisprofundo, elinstrumen to de recoleccióndedatosutilizadoenla presenteinvestigaciónresulta insuficien tepara res pon derestainterrogante, la cual puede seraborda da mejora través de his to rias de vida deungrupode es tudian tes seleccionadosdeformaaleatoria (a findega rantizarlarepresentatividaddelos resultados), con un ade cua do con trol de va ria bles (por ejem plo, la edad deini cio en el uso de consolas para videojuegos).Ellopermi tirálo grar unnivel de rigu rosidad acep table en el análisis del condicionamiento so cialcomo va riableexplicativa delas diferenciasencon tradascon relaciónalaconductadeusodeInternetsegúnelsexodelosusuarios.

\section{CONCLUSIONES}

Enpromedio, las es tu dian tesuniversita rias usanInternetconmenor frecuenciay tienen menos destreza en el manejo de la red, a diferencia de los hombres, quienes exhiben mayor dominio de esta herramienta. Por otro lado ellos prefieren usar, en primerlugar, elcorreoelectrónico, mien tras quelos muje res muestransupreferencia por el World Wide Web. La preferencia masculina por el correo electrónico no es consis ten te con los re sul ta dos de es tu dios previos, los cua les mues tran mayoracogi da por partedel público femenino, bá sica men teporla po sibilidad que ofreceelcorreodeampliarlas re des so ciales yutilizarun es tilode comunica ción ex presivoy amical. Poresta ra zón se re co mien da ex plo rar con más pro fun di dad la rela ción en tre el uso del correo electrónico y el sexo del usuario de Internet. Con relación al World Wide Web, dado que se tratade unaherramientaqueofrece contenidobásicamente textual, es comprensible la preferencia mayoritaria de las estudiantes, quienes, en promedio, tienenmejoreshabilidadeslectorasquelosuniversitarios.

Sobre la conducta de uso de Internet, las universitarias encuestadas consideran que tie nen poco do mi nio de la he rra mien ta ylos va ro nes se per ciben como co no cedo res de In ternet. Aun cuan do se en con tra ron di fe ren cias se gún el sexo, tan to en el patróndeuso comoensuopiniónsobreInternet, és tasnoresultaronsignificativas ( $p$ $>0.05$ ). No obstante, esos resultados son similares a los hallazgos de estudios previos, dondeseencontrarondiferenciassexualesimportantes.

Estas diferencias en los patrones de uso de Internet y, en general, en el manejo de computado ras, pue denabordarse tenien doencuentaelestilo deapren diza je dehombres y mu jeres. Por ejem plo, en lu gar de de sa rro llar las cla ses de tec no lo gía de lain formación sobre la base de demostraciones visuales (e.g., mostrar los tutoriales de cada temautilizan doun proyectormultimedia), las cuáles refuerzanlashabilidadesviso-espaciales su pe rio res delos va rones, se pue de comple men tarla pro yecc ción delos tu toriales conla repe tición delas in trucciones envozalta.Deesta manera, las universitarias aprovecharánsuren dimiento superioren el procesamien to depalabrasenparticular y 
en las ta reas verba les engene ral, he cho que ha sido de mos tra do en es tu dios previos, citadosenlaprimerapartedeesteartículo.

Las dife renciasobservadaspuedenexplicarsepordoshechosqueseobservan frecuentemente: la participación en cursos de capacitación de herramientas Internet e in te rés por los video jue gos. Los cursos de ca pa ci ta ción per miten re du cir la curva de aprendizaje en el manejo de Internet, mientras que los videojuegos y los juegos por computadora suelen preparar a los adolescentes en el manejo experto de Internet. No es ca sual que el au tor haya ob serva do que una de las for mas más efec ti vas de iniciarse enInternetes bus carin forma ción so brelosvideojuegos: manuales, trucos, etcétera Por todo ello en investigaciones futuras se recomienda incluir preguntas que explo ren lacapacita ción previa en el manejo de herra mien tas In ternetylaexperiencia con juegos por computadora, terreno don de la bre cha en tre hom bres y muje res prácticamentehadesaparecido, al menos en el merca do nor te ame rica no. De acuer docon unin formepublicadoporla Asocia ción deProgramasDigitales Inte rac tivos en elmes de abril de 2002, las mu je res cons ti tuían el $50 \%$ de los con su mi do res de jue gos elec trónicos en los Es ta dos Uni dos y el $48 \%$ de ellashabía compra docon so las. Y este ingreso masivo de mujeres al mercado de los videojuegos y las consolas no se daba únicamente en las series de juegos de Barbie, sino también en juegos como el SimCity,consideradoeljuegomásvendidoparacomputadoraspersonales.

Finalmente, se debe señalarquelas diferenciasdees tos re sul tados conlosobtenidos porFord y Miller $(1996,2001)$ pue den deberse al tipo de po bla ción es tu dia da, ya que ellosin cluyeron en sumues tra es tu dian tes de postgrado, mien tras quela mues tra del presen te es tu dio fue con alum nos ma tri cu la dos en cur sos de pregra do. Esto supone una aproximación di fe ren te a In ter net por que a ni vel de pre gra do no se pone tan toén fasisenelde sarrollo dehabilida desparalabúsquedayrecuperacióndeinformaciónusan dobases de da tosy mo to res de bús que da disponibles en In ternet, como sí sucede en los cursos de postgrado, probablemente porque el estudiante de postgrado ya ha desarrollado esas habilidades a lo largo de su formación profesional o porque la can ti dad dema tricula dos en es tos cursos per mi te brin darun servicio personalizadoynomasificado, comomuchasvecesocurreenelniveldepregrado.

Enese sentido, seríain teresan te quelos programas de educa ción de usuariosanivel uni ver si ta rio in clu ye ran no sólo el ma nejo de las ba ses de da tos y delos re cursos dela biblioteca(léa se, libros, re vis tas, dis coscompactos, vídeos), sino tam biénelde sarrollo de habilidades básicas que le permitan a los estudiantes aprender de forma independiente elsabermanejar los re cursos dein for ma ción sinverse des borda dos porsugran volumen. Al res pecto, la aplica ción de programas de capa cita ción ba sa dos enla re solución deproblemas, dentrodeuncontex tocaracteriza do porelusodelastecnologías de la información, halogradomejo ras significativas en eldesempeñoaca démicodelosestudiantes, hombres y mujeres (Eisenberg y Berkowitz, 1990; Herring, 1997). Si bien es cier to quelas pro pues tas más exi to sas-como el mo deloPLUSy el en fo que de los Seis Grandes- han sido usadas principalmente en colegios, estos enfoques tienen la suficienteflexibilidad paraadaptarsea diversoscontex tos educa tivos. Porejemplo, en las 
universida des se podría empezarcon programas decapacita ción para desarrollarha bilidadesenlabúsquedadeinformación, dirigidosalos pro fesoresuniversitarios y, posteriormen te, cola bo rarcon ellos a fin dein tro du cir es tos pro gra mas den tro dela programación curriculardeloscursos (porejemplo,Metodologíadelainves tiga ción, Recursos deinformación, etcétera). Entérminos pedagógicos estose ex presaría en planes declase y unida des didác ti cas, así como en ma trices de eva lua ción y for ma tos dellena do rá pido. Deestaformaselograríaunimportanteefectomultiplicador.

\section{REFERENCIAS}

Apoyo Opinión y Mercado (2002). Usos y actitudeshacia Internet 2002. Lima : Apoyo Opinión y Mercado.

Arnold, A. (1980). Sexual differences in the brain. American Scientific, 68, $165-173.7$

Bo ne va. B; Kraut, R. y Frohlich. D. (2001). Using E- mail for per so nal relationships: the difference gender makes. American Behavioral Scientist. Númeroespecialsobre "The In ter net and Everyday Life", 45 (3), 530549.

Bozionelos, N. (1995). Gender differences in attitudes towards computers with organizational and workplace implications. Occupational Psychologist, 26 de agosto de 1995, 3-5.

Bozionelos, N. (1996). Computer anxiety and negative attitudes towards computers: issues of no con cern for the fu tu re.BPS AnnualConference, $1-5$.

Brosnan, M. (1998). The impact of computer anxiety and self-efficacy upon performance. Journal of Computer Assisted Learning, 14, 223-234.

Eden, C. y Hul bert, W. (1995). Gen der and IT in the primary classroom: building confidence through laptops. Computer Education, 81, 10-14.

Eisenberg, M. Berkowitz, R. (1990). Information problem-solving: the big six approach to library and information skills instruction. Norwood, N. J.: Ablex.

Erwin, R.; Mawhinney-Hee, M.; Gur, R. C. y Gur, R. (1989). Effects of task and gender on EGG indices of hemispheric activation. Neuropsychiatry, Neuropsychology and Behavioral Neurology, 2 (4), 248-260.

Ford, N. y Miller, D. (1996). Gender differences in Internet perception and use. Aslib Proceedings, 48 (7/8), 183-192.

Ford, N.; Miller, D. y Moss, N. (2001). The role of individualdifferences in In ternet sear ching: an em pi rical study. Journal of the Ame rican Society of Information Science and Technology, 52 (12), 1049-1066. 
Gu ra in, M. y Hen ley, P. (2001). Boys and girls learn diffe rently: a gui de for teachers and parents. San Francisco: Jossey-Bass, 345 p.

Haier, R. y Benbow, C. (1995). Sex differences and lateralization in temporal lobe glucose metabolism during mathematical reasoning. Developmental Neuropsychology, 11 (4), 403-412.

Harris, R. (1999). Genderand te chnology rela tions in libra rianship. Journal of Education for Library and Information Science, 40 (4), 232-246.

Heimrath, R. y Goulding, A. (2001). In ter net per cep tion and use: a gen der perspective. Program, 15 (2), 119-134.

He rring, S. (1994). Gen der diffe ren ces in com pu ter- me dia ted commu ni cation: Bringing fa miliarbaggage to the new fron tier. Trabajopresentado en la "Convention of the American Library Association", Miami, USA. Disponible en http://www.cpsr.org/gender/herring.txt. [Consultado el 16 de noviembre de 2002].

He rring, S. (1996). Pos ting in a diffe rent voi ce: Gen der and ethics in computer-mediated communication. En: C. Ess (Ed.), Philosophical approaches to computer-mediated communication . Albany: SUNY Press, pp. 115-145.

Katz, J. y Aspden, P. (1997). Mo ti va tions for and ba rriers to In ter net usa ge: Re sults of a na tio nal pu blic survey opi nion. Internet Re search:Electronic Networking Applications and Policy, 7 (3), 170-188.

Kimu ra,D. (1987). Are men's and wo men's bra ins rea lly diffe rent?. Canadian Psychology, 28, 133-147.

Kimu ra, D. (1992). Sex diffe ren ces in the bra in.Scientific American, 267 (3), 118-125.

Kraut, R.; Scherlis, W.; Mukhopadhyay, T.; Manning, J. y Kiesler, S. (1996). The HomeNet field trial of residential Internet services. Communications of the ACM, 39, 55-63.

Leong, S. y Al-Hawamdeh, S. (1999). Gender and learning attitudes in using Web- ba sed scien ce lessons. Information Research, 5(1). Disponible en http://informationr.net/ir/5-1/paper66.html. [Consultado el 15 de noviembre de 2002]

Moir, A. y Jessel, D. (1991). Brain sex: the real difference between men and women. New York: Laurel.

Moir, A. y Moir, B. (2000). Why men don't iron: the fas ci na ting and unal terable differences between men and women. New York: Citadel, 314 p.

Morahan-Martin,J.,ySchuma cher,P.(1997, agos to). Genderdifferences in In ternetusa ge, beha viors, and atti tu des among un dergra dua tes. En: G. Sal vendy (Ed.), HCI In ter na tio nal '97: Proce edings of the 7 th In ternational Conference on Human-ComputerInteraction (p. 122). Amsterdam: Elsevier Science. 
Sa vicki, V.; Ke lley, M. y Lin gen fel ter, D. (1996). Gen der and group composition in small task groups using computer-mediated communication. Computer in Human Behavior, 12, 209-224.

Saykin, A. yotros (1995). Norma tiveneu ro psy chological test per formance: effects of age, education, gender and ethnicity. AppliedNeuropsycho $\log y, 2,79-88$.

Schumacher, P., y Morahan-Martin, J. (1998, agosto). Are Internet and computerattitudesandbehaviors related? Genderdifferences. Trabajo presen ta do en la "106th An nual Conven tion of the Ame ri can Psy chological Association”, San Francisco, USA.

Shaywitz, B. y otros (1995). Sex diffe ren ces in the func tio nal or ga nization of the brain for language. Nature, 373, 607-609.

Tar ling, E. (1997). Com pu ters in the class room: whe re are the girls. Harvard Education Letter Focus Series, Technology and Schools, 3, 14-23.

Watson, N. (1991). Nontrivial sex differences in throwing and intercepting:relationtopsychometrically-defined spatialfunctions. Personaland Individual Differences, 12, 375-385.

Wi tel son, S. (1976). Sex and single he misphe re: spe cia liza tion of the right hemisphere for the spatial proccessing. Science, 193, 425-427.

Witel son, S. (1989). Hand and sex diffe ren ces in the ish tu mus and genu of the human corpus callousum. Brain, 112, 799-835.

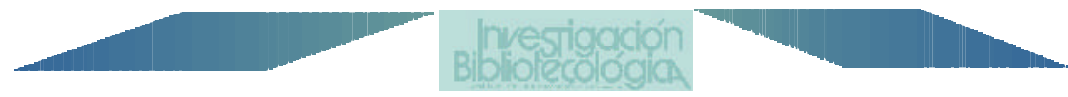

